\title{
Formation and characterization of furfuryl mercaptan- $\beta$-cyclodextrin inclusion complex and its thermal release characteristics
}

\author{
Guangyong Zhu', Zuobing Xiao",, Gengfa Yu",, Guangxu Zhu², Yunwei Niu, Junhua Liu \\ ${ }^{1}$ Shanghai Institute of Technology, No.100 Haiquan Road, Shanghai, 201418, PR China \\ ${ }^{2} 3035$ Sable Ridge Dr. Ottawa. ON K1T 3R9, Canada \\ "Corresponding authors: e-mail: zbingxiao@sina.com,genquan1@sina.com
}

\begin{abstract}
Furfuryl mercaptan has the aroma characteristics of coffee. However, it is unstable during storage of coffee brew and roasted coffee. In order to enhance the stability of furfuryl mercaptan, furfuryl mercaptan$\beta$-cyclodextrin inclusion complex was synthesized using the precipitation method in this work. Fourier transform infrared spectroscopy, x-ray diffraction, and thermogravimetric analysis (TG) were used to characterize the resulting products. The interaction of furfuryl mercaptan with $\beta$-cyclodextrin was investigated by the molecular mechanics (MM) method. These changes in FTIR and XRD gave supporting evidence for the successful formation of furfuryl mercaptan- $\beta$-cyclodextrin inclusion complex. The TG results showed that the formation of furfuryl mercaptan- $\beta$-cyclodextrin inclusion complex could improve the thermal stability of furfuryl mercaptan and provide a long-lasting effect. The structure of furfuryl mercaptan- $\beta$-cyclodextrin inclusion complex with the minimum energy was obtained by MM 2 calculation, and the minimum binding energy was $-77.0 \mathrm{~kJ} \mathrm{~mol}^{-1}$ at $-1.96 \times 10^{-10} \mathrm{~m}$.
\end{abstract}

Keywords: Furfuryl mercaptan- $\beta$-cyclodextrin, Inclusion complex, XRD, FTIR, Thermal analysis, Molecular mechanics.

\section{INTRODUCTION}

Furfuryl mercaptan (see Fig. 1), an important aroma constituent of flavor, was found in coffee, sesame seed oil, popcorn, grilled pork, cooked beef, and roasted chicken ${ }^{1}$. It has the aroma characteristics of coffee, sesame, onion, garlic, and meat. Furfuryl mercaptan has been widely used in baked goods, gelatins, alcoholic and nonalcoholic beverages, condiments, relishes, frozen dairy gravies, hard and softy candy, and meat products. Furfuryl mercaptan, which is soluble in oils but insoluble in water, is a colorless oily liquid with boiling point $154-155^{\circ} \mathrm{C}^{\mathbf{1}}$.

Furfuryl mercaptan exerts an important effect on coffee flavor profiles. It is a key ingredient of the characteristic coffee flavor. In coffee flavor field, the identification of furfuryl mercaptan was considered as a milestone ${ }^{2}$. However, because of physical volatile loss and chemical reaction, its odor quality is unstable. Due to its interaction with the melanoidins (which were formed during the Maillard reaction, e.g., coffee roasting), furfuryl mercaptan is unstable during storage of coffee brew and roasted coffee ${ }^{3}$. Melanoidins can promote the degradation of furfuryl mercaptan ${ }^{4}$. It was one of the major reasons for the sulfury-roasty odor quality decrease. When furfuryl mercaptan was added to water plus melanoidins, approximately $50 \%$ of furfuryl mercaptan was lost after $10 \mathrm{~min}$ of storage and it was nearly absent after $30 \mathrm{~min}$ of storage ${ }^{5}$. Through the reactive quinone converted from hydroxyhydroquinone, furfuryl mercaptan can also react with hydroxyhydroquinone $e^{3,6}$. Furthermore, furfuryl mercaptan is unstable and tends to polymerize when it was heated in the presence of mineral acids ${ }^{3,6}$.

The reactions of furfuryl mercaptan and physical volatile loss are the main reasons for loss of the aroma

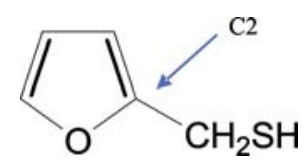

Figure 1. Furfuryl mercaptan chemical structure formula of coffee and affect the quality and stability of coffee aroma, which has become a problem that plagues the industry ${ }^{7}$. How to reduce the loss of furfuryl mercaptan, keep its aroma stable, and improve the aroma quality of coffee, has become an urgent problem to be solved in the industry. How to effectively enhance the stability of furfuryl mercaptan, control its release, improve its aroma retention, and enhance its water solubility is of great significance.

Core materials can be encapsulated in wall materials by formation of inclusion complex. It can realize the protection of core materials and improve their stability. Therefore, by encapsulation of furfuryl mercaptan to form inclusion complex, it is possible to realize the protection of furfuryl mercaptan and enhance its stability. Among many wall materials, $\beta$-cyclodextrin, as a safe and environmentally friendly wall material, has attracted much attention and becomes a research hotspot ${ }^{8,9} \cdot \beta$-cyclodextrin molecule has a slightly tapered hollow cylinder three-dimensional ring structure formed by glucopyranose units. Its outer surface has hydrophilic characteristics, while the inside of the molecule has hydrophobic characteristics because of the $\mathrm{C}-\mathrm{H}$ bond shielding effect. Various organic molecules can be encapsulated in its hydrophobic cavity to form inclusion complexes and change the physicochemical properties of these entrapped molecules ${ }^{10}$. In most cases, inclusion complex formation with $\beta$-cyclodextrin can improve stability of poorly stable substances ${ }^{11}$. Pires et al. found that the shelf-life increase achieved for thymol standard was $354 \%$ and Lippia origanoides essential oil had a stability increase of about $45 \%{ }^{12}$. Ikeda et al. found that water solubility of anionic nateglinide could be improved by complexation of $\beta$-cyclodextrin ${ }^{13}$.

Furfuryl mercaptan- $\beta$-cyclodextrin inclusion complex was synthesized using the precipitation method in this work to improve the stability of furfuryl mercaptan, control its release, improve its aroma retention, and 
enhance its water solubility. Fourier transform infrared (FTIR) spectroscopy, thermogravimetric (TG) analysis, and $\mathrm{x}$-ray diffraction (XRD) were used to characterize the resulting products. The formation of furfuryl mercaptan$\beta$-cyclodextrin inclusion complex was investigated by the molecular mechanics (MM) method at the molecular level. Binding energy calculation and structure optimization were performed with MM2.

\section{EXPERIMENTAL}

\section{Materials}

Furfuryl mercaptan (food grade, $\mathrm{C}_{5} \mathrm{H}_{6} \mathrm{OS}$, molecular weight 114, colorless oily liquid) was purchased from Guangzhou Levon Flavor \& Fragrance Technology Co., Ltd. Anhydrous ethanol was of analytical grade and was provided by Shanghai Sinopharm Chemical Reagent Co., Ltd. Deionized water adopted throughout the experiments was produced in our laboratory. $\beta$-cyclodextrin $\left(\mathrm{C}_{42} \mathrm{H}_{70} \mathrm{O}_{35}\right.$, molecular weight 1134 , white crystalline powder) was of pharmaceutical grade and was purchased from Shandong Binzhou Zhiyuan Bio-Technology Co., Ltd. Without out further purification, all the raw materials were used directly in the experiment.

\section{Methods}

Formation of furfuryl mercaptan- $\beta$-cyclodextrin inclusion complex

With some modifications, the precipitation method as described in references was used to prepare furfuryl mercaptan- $\beta$-cyclodextrin inclusion complex ${ }^{14,15}$. Firstly, $5 \mathrm{~g}$ of $\beta$-cyclodextrin was added to $93 \mathrm{~g}$ of deionized water and was stirred to form a suspension. The temperature of the suspension was kept at $35^{\circ} \mathrm{C}$. Then, $2 \mathrm{~g}$ furfuryl mercaptan was slowly added to this suspension. Excess furfuryl mercaptan was used in the experiment. Such molar ratio of furfuryl mercaptan to $\beta$-cyclodextrin was used to ensure the cavities of $\beta$-cyclodextrin molecules containing furfuryl mercaptan molecules. After the addition of furfuryl, the temperature of the suspension remained at $35{ }^{\circ} \mathrm{C}$ and the mixture was continuously stirred for $3 \mathrm{~h}$ to form furfuryl mercaptan- $\beta$-cyclodextrin inclusion. The suspension was stored in a refrigerator at $5{ }^{\circ} \mathrm{C}$ overnight. The precipitate was obtained with the vacuum filtration method. Anhydrous ethanol was used to wash the precipitate. After drying in a freeze drier (FD-1A-50), the product was kept in a desiccator for further analysis.

\section{Characterization by FTIR}

A Vertex 70 Fourier transform infrared spectrometer (Bruker, Germany) was adopted to determine the FTIR spectra of furfuryl mercaptan, $\beta$-cyclodextrin and furfuryl mercaptan- $\beta$-cyclodextrin inclusion complex. The frequency range was $400-4000 \mathrm{~cm}^{-1}$.

\section{Characterization by XRD}

A D/Max 2000X X-ray diffractometer (Rigaku Corporation, Japan) was used to determine $\mathrm{x}$-ray diffraction of the $\beta$-cyclodextrin and furfuryl mercaptan- $\beta$-cyclodextrin inclusion complex as described in references ${ }^{\mathbf{1 4}, 16}$. At 100
$\mathrm{mA}$ and $40 \mathrm{kV}, \mathrm{Cu} \mathrm{K} \alpha$ radiation was adopted. The $2 \theta$ range was $5^{\circ}$ to $70^{\circ}$.

Characterization of furfuryl mercaptan release from its inclusion complex by thermogravimetric (TG) analysis

The rate of mass loss and mass loss of samples were performed with a TGA-Q5000IR thermogravimetric analyzer (TA Instruments, USA). About $5 \mathrm{mg}$ of $\beta$-cyclodextrin or furfuryl mercaptan- $\beta$-cyclodextrin inclusion complex in a ceramic crucible was weighed. The heating rate adopted in the pyrolysis process was $10{ }^{\circ} \mathrm{C} \mathrm{min}-1$. High purity nitrogen gas was used to avoid oxidation during the pyrolysis process and the gas flow was $20 \mathrm{ml} \mathrm{min}$.

\section{Binding energy calculation and structure optimization by molecular mechanics (MM) calculations}

MM2 calculations were adopted to examine furfuryl mercaptan- $\beta$-cyclodextrin inclusion complex formation at the molecular level. Binding energy calculation and structure optimization were carried out by Chem3D Ultra (CambridgeSoft Corporation, MA, USA). The process of encapsulation of furfuryl mercaptan in $\beta$-cyclodextrin was simulated by successively changing the $\mathrm{Z}$ coordinate of the furfuryl mercaptan atoms after properly orientating $\beta$-cyclodextrin and furfuryl mercaptan molecules. The docking strategy as described in references was used, i.e. push furfuryl mercaptan molecule stepwise through the $\beta$-cyclodextrin orifice minimizing the energy of the complex at each step ${ }^{\mathbf{1 5}} \mathbf{1 7}$. The position of furfuryl mercaptan molecule relative to $\beta$-cyclodextrin molecule was referred to the $\mathrm{Z}$ coordinate of $\mathrm{C} 2$ atom of furfuryl mercaptan. Furfuryl mercaptan moving direction during MM2 calculation is shown in Fig. 2.

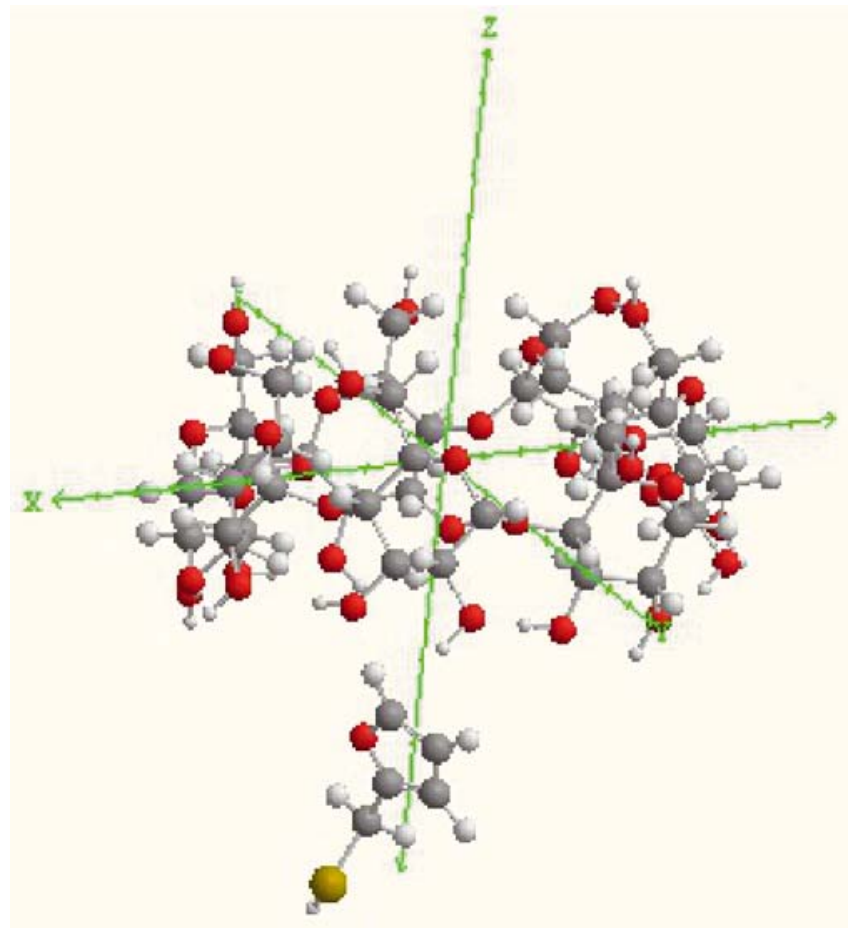

Figure 2. Furfuryl mercaptan moving direction during MM2 calculation 


\section{RESULTS AND DISCUSSIONS}

The results of FTIR of furfuryl mercaptan, $\beta$-cyclodextrin and furfuryl mercaptan- $\beta$-cyclodextrin inclusion complex

Fig. 3 shows the FTIR curves of furfuryl mercaptan, $\beta$-cyclodextrin and furfuryl mercaptan- $\beta$-cyclodextrin inclusion complex. The obvious FTIR peaks of $\beta$-cyclodextrin appear at $3356 \mathrm{~cm}^{-1}, 2922 \mathrm{~cm}^{-1}, 1643 \mathrm{~cm}^{-1}, 1404 \mathrm{~cm}^{-1}$, $1244 \mathrm{~cm}^{-1}, 1151 \mathrm{~cm}^{-1}, 1030 \mathrm{~cm}^{-1}, 937 \mathrm{~cm}^{-1}$ and $848 \mathrm{~cm}^{-1}$. As shown in Fig. 3, except for some minor changes in peak position, the FTIR curves of $\beta$-cyclodextrin and furfuryl mercaptan- $\beta$-cyclodextrin inclusion complex have a similar shape. The broad peaks appear at $3356 \mathrm{~cm}^{-1}$ and $3337 \mathrm{~cm}^{-1}$ in the FTIR curves of $\beta$-cyclodextrin and furfuryl mercaptan- $\beta$-cyclodextrin inclusion complex respectively can be attributed to stretching $(\mathrm{O}-\mathrm{H})$ vibration of hydroxyl groups in $\beta$-cyclodextrin molecule ${ }^{\mathbf{1 5}}$. After the formation of furfuryl mercaptan- $\beta$-cyclodextrin inclusion complex, the peak caused by the stretching vibration of hydroxy groups moved towards the low band and blue-shift was observed. The strong sharp peaks at $1030 \mathrm{~cm}^{-1}$ and $1032 \mathrm{~cm}^{-1}$ in the FTIR curves of $\beta$-cyclodextrin and furfuryl mercaptan- $\beta$-cyclodextrin inclusion complex respectively can be assigned to stretching (C-O) vibration. However, after the formation of the inclusion complex, the peak moved towards the high band and red-shift was observed. The shoulder peak at $1151 \mathrm{~cm}^{-1}$ did not change before and after the formation of furfuryl mercaptan- $\beta$ cyclodextrin inclusion complex. The weak peak appearing at $2565 \mathrm{~cm}^{-1}$ in the FTIR curve of furfuryl mercaptan is due to stretching $(\mathrm{S}-\mathrm{H})$ vibration ${ }^{18}$. Owing to furan ring stretching $(\mathrm{C}-\mathrm{H})$ vibration, a weak peak occurs at $3123 \mathrm{~cm}^{-1}$. Because of the furan ring stretching $(\mathrm{C}=\mathrm{C})$ vibration, three peaks occur at 1597,1501 and $1420 \mathrm{~cm}^{-1}$ respectively in the FTIR curve of furfuryl mercaptan. The peaks at 1254, 1151 and $1012 \mathrm{~cm}^{-1}$ can be assigned to furan ring asymmetrical stretching (C-O-C) vibration ${ }^{19-21}$. The strong sharp peak that occurs at $735 \mathrm{~cm}^{-1}$ is due to the out-of-plane bending $(\mathrm{C}-\mathrm{H})$ vibration of furan ring. In addition to the small peaks of furfuryl mercaptan at $3123,2526,1597,1510$ and $1420 \mathrm{~cm}^{-1}$, these characteri-

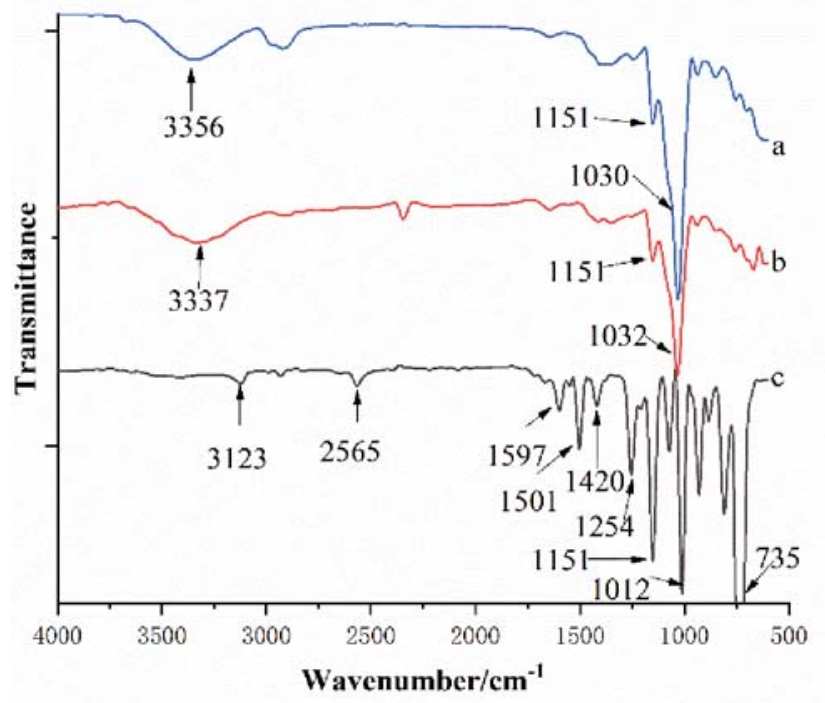

Figure 3. The FTIR spectra of $\beta$-cyclodextrin (a), furfuryl mercaptan- $\beta$-cyclodextrin inclusion complex (b), and furfuryl mercaptan (c) stic strong peaks of furfuryl mercaptan such as at 1012 and $735 \mathrm{~cm}^{-1}$ disappear as shown in the FTIR spectra of furfuryl mercaptan- $\beta$-cyclodextrin inclusion complex. The changes and the blue-shifting hydrogen bond of $\beta$-cyclodextrin after interaction with furfuryl mercaptan give evidence of successful encapsulation of furfuryl mercaptan in $\beta$-cyclodextrin.

The results of XRD of $\beta$-cyclodextrin and furfuryl mercaptan- $\beta$-cyclodextrin inclusion complex

As an effective instrument, XRD can be used to determine the formation of furfuryl mercaptan- $\beta$-cyclodextrin inclusion complex ${ }^{\mathbf{1 6}}$. The XRD peaks of $\beta$-cyclodextrin will change after the formation of the furfuryl mercaptan inclusion complex. The XRD curves of the furfuryl mercaptan- $\beta$-cyclodextrin inclusion complex and $\beta$-cyclodextrin are shown in Fig. 4.

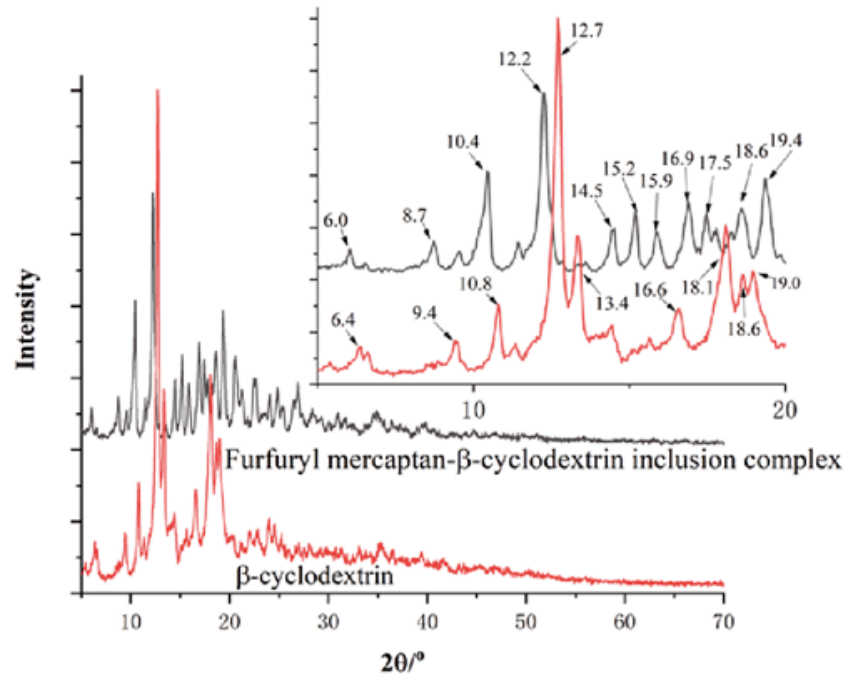

Figure 4. The XRD curves of $\beta$-cyclodextrin and furfuryl mercaptan- $\beta$-cyclodextrin inclusion complex

In the XRD curve of $\beta$-cyclodextrin, a strong peak appears at $12.7^{\circ}$ as shown in Fig. 4. However, in the XRD curve of the furfuryl mercaptan- $\beta$-cyclodextrin inclusion complex, this peak shifts to $12.2^{\circ}$. The peak intensity decrease can also be observed. Furthermore, the peaks at $10.8,9.4$, and $6.4^{\circ}$ in the XRD curve of $\beta$-cyclodextrin also shift to lower $2 \theta$ angles of 10.4 , 8.7 , and $6.0^{\circ}$ respectively in the XRD curve of furfuryl mercaptan- $\beta$-cyclodextrin inclusion complex. A similar phenomenon for peak shift was also found in the XRD patterns of mentha- 8 -thiol-3-one- $\beta$-cyclodextrin inclusion complex and menthyl acetate- $\beta$-cyclodextrin inclusion complex $^{14,22}$. The increase in the intensity of the peak at $10.4^{\circ}$ in the XRD pattern of $\beta$-cyclodextrin can be observed compared to the peak at $10.8^{\circ}$. However, the peaks at 16.6 and $19.0^{\circ}$ shift to a higher $2 \theta$ angle of 16.9 and $19.4^{\circ}$, respectively, and the peaks at 13.4 and $18.1^{\circ}$ disappear after the interaction of $\beta$-cyclodextrin and furfuryl mercaptan. Compared with $\beta$-cyclodextrin, some new peaks appear at $14.5,15.2,15.9$ and $17.5^{\circ}$ in the XRD curve of furfuryl mercaptan- $\beta$-cyclodextrin inclusion complex. The encapsulation of furfuryl mercaptan molecule in the cavity of $\beta$-cyclodextrin molecule may cause the XRD peaks shifting and the new ones appearing in the complex. Like the results of FTIR, these changes in XRD further give another supporting evidence for the 
successful formation of furfuryl mercaptan- $\beta$-cyclodextrin inclusion complex.

\section{Furfuryl mercaptan thermal release characteristics from furfuryl mercaptan- $\beta$-cyclodextrin inclusion complex}

Thermal analysis is another effective method of investigation of the interaction between host and guest molecules. The inclusion compound stoichiometry can also be evaluated by thermal analysis. Therefore, thermal analysis was adopted in the experiment to study the interaction between furfuryl mercaptan and $\beta$-cyclodextrin. The rate of mass loss and mass loss curves of $\beta$-cyclodextrin and furfuryl mercaptan- $\beta$-cyclodextrin inclusion complex obtained are shown in Fig. 5. There are three main stages in the mass loss curves of both $\beta$-cyclodextrin and furfuryl mercaptan- $\beta$-cyclodextrin inclusion complex as shown in Fig. 5. The first stage refers to the temperature range from room temperature to $290.6^{\circ} \mathrm{C}$. In the first stage, a slightly mass loss can be observed. The second stage refers to the temperature range from $290.6{ }^{\circ} \mathrm{C}$ to $350{ }^{\circ} \mathrm{C}$. In the second stage, the major mass loss occurred. The third stage refers to the temperature range from $350{ }^{\circ} \mathrm{C}$ to $500{ }^{\circ} \mathrm{C}$. In the third stage, a slightly mass loss occurred again.

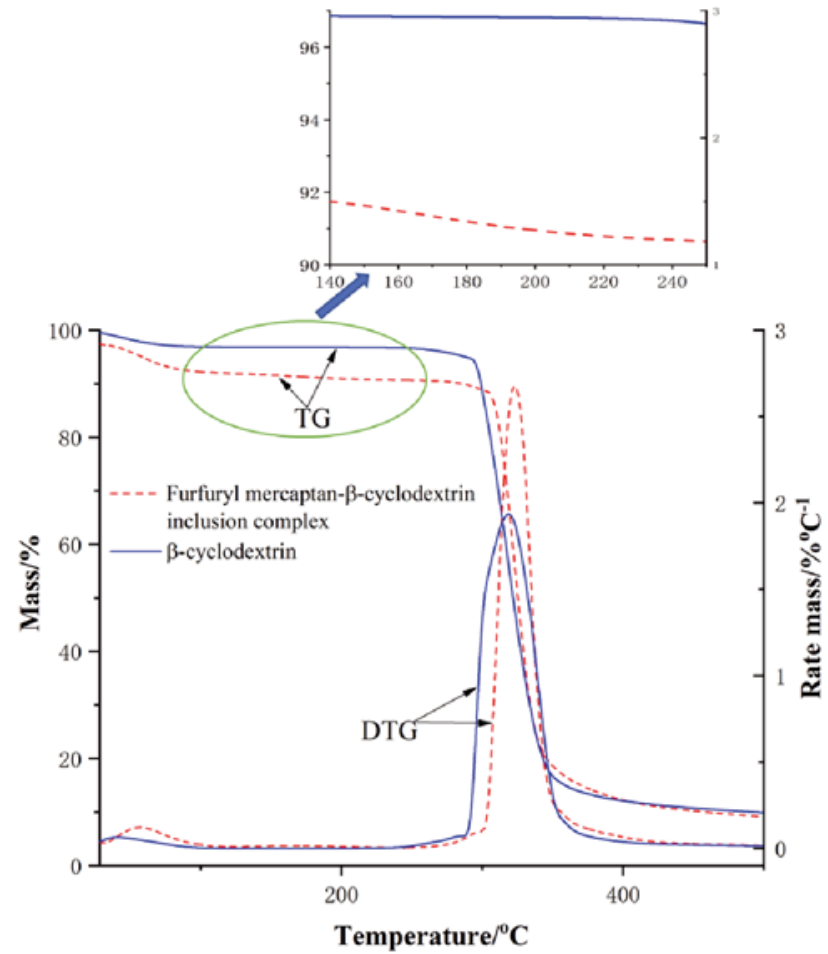

Figure 5. The TG and DTG curves of $\beta$-cyclodextrin and furfuryl mercaptan- $\beta$-cyclodextrin inclusion complex

In the TG curve of $\beta$-cyclodextrin, the slightly mass loss occurring in the first stage can be mainly attributed to desorption water; while in the mass loss curve of furfuryl mercaptan- $\beta$-cyclodextrin inclusion complex, the release of furfuryl mercaptan caused the main mass loss occurring in the first stage. In the temperature range of 140 to $250{ }^{\circ} \mathrm{C}$, the TG curve of $\beta$-cyclodextrin is leveling off while the mass loss curve of furfuryl mercaptan- $\beta$ cyclodextrin is downward sloping. The difference can be attributed to the release of furfuryl mercaptan from furfuryl mercaptan- $\beta$-cyclodextrin inclusion complex. Furfuryl mercaptan is soluble in oils and insoluble in water. During the inclusion process, all the water mole- cules might be expelled from the hydrophobic cavities of $\beta$-cyclodextrin molecules ${ }^{17}$. So, the water that is included furfuryl mercaptan- $\beta$-cyclodextrin inclusion complex can be ignored. Therefore, in the mass loss curve of furfuryl mercaptan- $\beta$-cyclodextrin inclusion complex, the mass loss occurring in the first stage may be explained simply as caused by the release of furfuryl mercaptan. In the first stage, the mass loss of furfuryl mercaptan- $\beta$-cyclodextrin inclusion complex is approximately $8 \%$. Therefore, the loading capacity of furfuryl mercaptan, which is defined as the mass ratio of furfuryl mercaptan to furfuryl mercaptan- $\beta$-cyclodextrin inclusion complex, is about $8 \%$. The molecular weights of furfuryl mercaptan and $\beta$-cyclodextrin are 114 and 1134 respectively. Therefore, the molar ratio of furfuryl mercaptan to $\beta$-cyclodextrin obtained from the mass loss is approximately 0.9:1. If guest:host stoichiometry is considered as $1: 1$, the theoretical loading capacity of furfuryl mercaptan is $9 \%$. Therefore, the furfuryl mercaptan: $\beta$-cyclodextrin stoichiometry is close to $1: 1$.

Because of the thermal decomposition of $\beta$-cyclodextrin, major mass loss can be observed in the second stage. Two strong peaks are appearing in the rate mass loss curves of both $\beta$-cyclodextrin and furfuryl mercaptan- $\beta$-cyclodextrin inclusion complex. In the third stage, a slightly mass loss occurred again because of the continuous decomposition of solid residuals of $\beta$-cyclodextrin at a very slow rate with the increase of temperature.

Because the boiling point of furfuryl mercaptan is $154-155^{\circ} \mathrm{C}$, furfuryl mercaptan should evaporate away completely before it reaches the boiling point during the pyrolysis process. However, the mass loss still occurred from $155 \mathrm{C}$ to the decomposition temperature of $\beta$-cyclodextrin. It can be observed obviously from the TG curve of furfuryl mercaptan- $\beta$-cyclodextrin inclusion complex in this stage. During the pyrolysis process, the encapsulated furfuryl mercaptan was gradually released from its inclusion complex. It further demonstrates the successful encapsulation of furfuryl mercaptan in $\beta$-cyclodextrin. By the formation of furfuryl mercaptan- $\beta$ cyclodextrin inclusion complex, long-lasting effect can be provided and the thermal stability of furfuryl mercaptan can be improved.

\section{The results of molecular mechanics calculations}

Binding energy is defined as the difference between the total energy of a furfuryl mercaptan- $\beta$-cyclodextrin inclusion complex molecule and the sum of the total energy of furfuryl mercaptan and $\beta$-cyclodextrin molecules. In other words, binding energy can be used to measure the energy required to break up a host-guest inclusion complex molecule completely into its host molecule and guest molecule. To some extent, the combination and the interaction between $\beta$-cyclodextrin and furfuryl mercaptan can be reflected by binding energy. Fig. 6 shows the plot of binding energy vs. the $\mathrm{Z}$ coordinate of $\mathrm{C} 2$ of furfuryl mercaptan molecule.

During the development of the furfuryl mercaptan- $\beta$ cyclodextrin inclusion complex, energy is released, so the value of binding energy is negative. When the $\mathrm{Z}$ coordinate of $\mathrm{C} 2$ increases from $-21.4 \times 10^{-10} \mathrm{~m}$ to $-10.9 \times 10^{-10} \mathrm{~m}$, the binding energies change slightly as shown in Fig. 6 . When $\mathrm{Z}$ coordinate increases from $-10.9 \times 10^{-10} \mathrm{~m}$, a sharp 


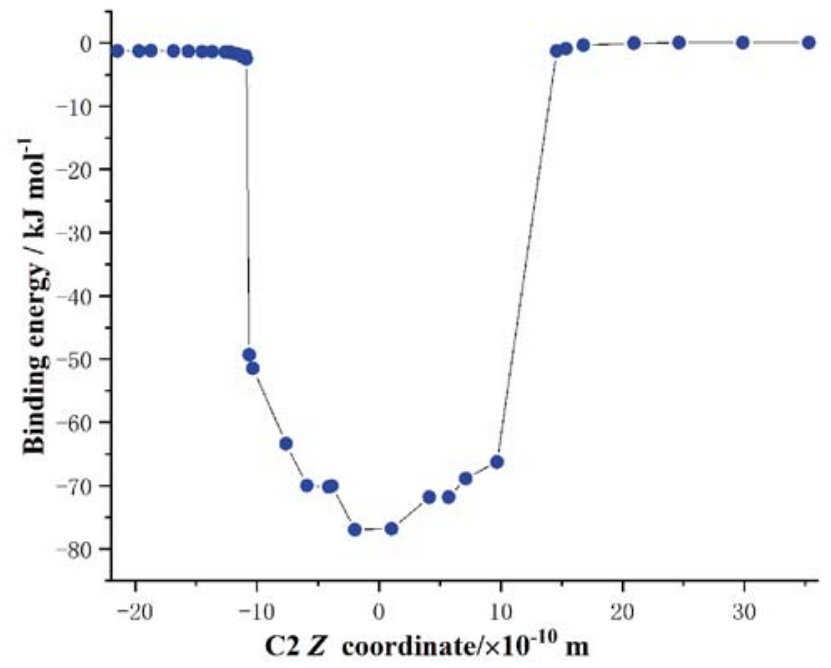

Figure 6. Plot of binding energy vs. the $\mathrm{Z}$ coordinate of $\mathrm{C} 2$ in furfuryl mercaptan molecule

fall in the value of binding energy can be observed. At $-10.6 \times 10^{-10} \mathrm{~m}$, the value of binding energy is $-49.3 \mathrm{~kJ}$ $\mathrm{mol}^{-1}$. With the increase of $\mathrm{Z}$ coordinate of $\mathrm{C} 2$ to -1.96 $\times 10^{-10} \mathrm{~m}$, the minimum value of binding energy, -77.0 $\mathrm{kJ} \mathrm{mol}^{-1}$, was obtained. When the $\mathrm{Z}$ coordinate of $\mathrm{C} 2$ changes from $-1.96 \times 10^{-10} \mathrm{~m}$ to $9.7 \times 10^{-10} \mathrm{~m}$, binding energy has an increasing trend. With a further increase of $\mathrm{Z}$ coordinate from $9.7 \times 10^{-10} \mathrm{~m}$ to $14.6 \times 10^{-10} \mathrm{~m}$, the binding energy rises sharply. When the $\mathrm{Z}$ coordinate of $\mathrm{C} 2$ changes from $14.6 \times 10^{-10} \mathrm{~m}$ to $35.3 \times 10^{-10} \mathrm{~m}$, the values of binding energy keep almost unchanged.

The more energy is released during the complexation process, the smaller the binding energy is, and the more stable the furfuryl mercaptan- $\beta$-cyclodextrin inclusion complex becomes. The binding energy for the most stable furfuryl mercaptan- $\beta$-cyclodextrin inclusion complex is $-77.0 \mathrm{~kJ} \mathrm{~mol}^{-1}$ at $-1.96 \times 10^{-10} \mathrm{~m}$. By MM2 calculation, geranial-monochlorotriazinyl- $\beta$-cyclodextrin, difurfuryl disulfide- $\beta$ - cyclodextrin, neral-momochlorotriaziny- $\beta$ cyclodextrin, and menthol-HP- $\beta$-cyclodextrin inclusion complex were previously investigated and the calculated binging energy values were $-135.2,-162,-143,-127 \mathrm{~kJ}$ mol $^{-1}$ respectively ${ }^{15,17,23}$. Compared with these previous values, $-77.0 \mathrm{~kJ} \mathrm{~mol}^{-1}$ is the largest binding energy. It means that furfuryl mercaptan- $\beta$-cyclodextrin inclusion complex is relatively unstable compared with difurfuryl disulfide- $\beta-C D$, neral-momochlorotriaziny- $\beta$-cyclodextrin, geranial-monochlorotriazinyl- $\beta$-cyclodextrin and mentholHP- $\beta$-cyclodextrin inclusion complex.

In the process of MM2 calculation, the total energy consists of 1,4 van der Waals, bend, non-1,4 van der Waals, stretch, stretch-bend, dipole/dipole, and torsion energy. The minimum binding energy consists of the following components: 1,4 van der Waals $\left(4.2 \mathrm{~kJ} \mathrm{~mol}^{-1}\right)$, bend $(-2.5 \mathrm{~kJ}$ $\left.\mathrm{mol}^{-1}\right)$, non-1,4 van der Waals $\left(-75.4 \mathrm{~kJ} \mathrm{~mol}^{-1}\right)$, stretch $(-1.4$ $\left.\mathrm{kJ} \mathrm{mol}^{-1}\right)$, stretch-bend $\left(-0.6 \mathrm{~kJ} \mathrm{~mol}^{-1}\right)$, dipole/dipole $(-0.4$ $\left.\mathrm{kJ} \mathrm{mol}^{-1}\right)$, and torsion energy $\left(-0.8 \mathrm{~kJ} \mathrm{~mol}^{-1}\right)$. Non- 1,4 van der Waals energy contributes a lot to binding energy, and is the main reason for the stability of furfuryl mercaptan$\beta$-cyclodextrin inclusion complex. Once furfuryl mercaptan molecule entered the cavity of $\beta$-cyclodextrin molecule, $\beta$-cyclodextrin changed its shape and furfuryl mercaptan molecule also made conformation adjustments to maximize the stabilization. The complexation geometries of furfuryl mercaptan molecule and $\beta$-cyclodextrin were constantly readjusted until the most stable inclusion complexes were obtained. By MM2 calculation, the obtained structure of furfuryl mercaptan- $\beta$-cyclodextrin inclusion complex which has the minimum energy is shown in Fig. 7. It is a relatively stable structure after shape and conformation adjustments of furfuryl mercaptan and $\beta$-cyclodextrin molecules during the process of MM2 calculation.

\section{CONCLUSIONS}

Furfuryl mercaptan- $\beta$-cyclodextrin inclusion complex was successfully synthesized in this work. Some characteristic peaks of furfuryl mercaptan disappeared in the FTIR spectra of furfuryl mercaptan- $\beta$-cyclodextrin inclusion complex, and blue-shifting hydrogen bond of $\beta$-cyclodextrin occurred after interaction with furfuryl mercaptan. The peaks at 12.7, 10.8, 9.4 and $6.4^{\circ}$ in the XRD curve of $\beta$-cyclodextrin shifted to lower $2 \theta$ angles of $12.2,10.4,8.7$, and $6.0^{\circ}$ respectively in the XRD curve of furfuryl mercaptan- $\beta$-cyclodextrin inclusion complex. These changes in FTIR and XRD gave supporting evidence for the successful formation of furfuryl mercaptan$\beta$-cyclodextrin inclusion complex. The boiling point of furfuryl mercaptan is $154-155{ }^{\circ} \mathrm{C}$, while the mass loss still occurred from furfuryl mercaptan- $\beta$-cyclodextrin inclusion complex in the temperature range of $155^{\circ} \mathrm{C}$ to the decomposition temperature of $\beta$-cyclodextrin.
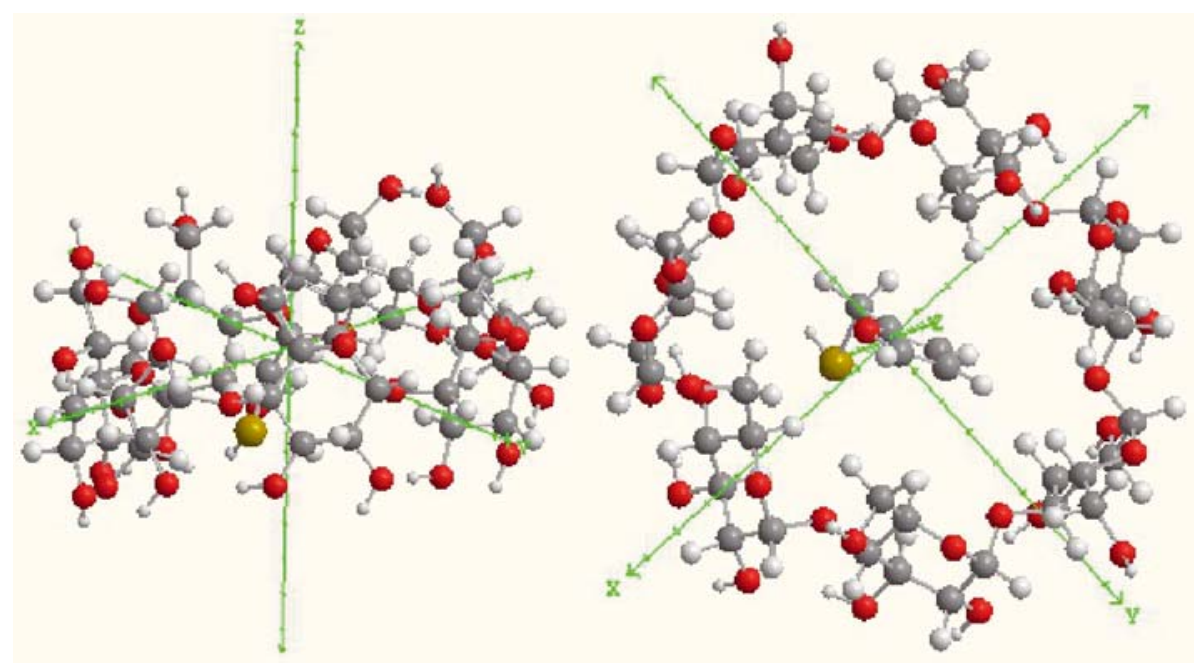

Figure 7. The MM2-computed structure of furfuryl mercaptan- $\beta$-cyclodextrin with the minimum energy 
It further demonstrated that the encapsulation of furfuryl mercaptan in $\beta$-cyclodextrin was successful and that long-lasting effect and thermal stability of furfuryl mercaptan were improved. Using MM2 calculation, the structure of furfuryl mercaptan- $\beta$-cyclodextrin inclusion complex was optimized and the minimum binding energy was calculated. This data is helpful to understand the interaction of furfuryl mercaptan and $\beta$-cyclodextrin. Encapsulation of furfuryl mercaptan by the formation of inclusion complex is a possible way to enhance the stability of furfuryl mercaptan, control its release, improve its aroma retention, and enhance its water solubility. Furfuryl mercaptan- $\beta$-cyclodextrin inclusion complex can be widely used in flavor, fragrance and food industries.

\section{ACKNOWLEDGMENTS}

The authors thank the financial supports provided by the National Key R\&D Program of China (2016YFA0200300), National Natural Science Found of China (31972196), Shanghai Alliance Program (LM201844), and Gaofeng \& Gaoyuan Project for University Academic Program Development.

\section{LITERATURE CITED}

1. Burdock, G.A. (2010). Fenaroli's handbook of flavor ingredients (6th ed.). Boca Raton, USA: CRC Press.

2. Sun, Z., Hayat, K., Yu, J., Karangwa, E., Duhoranimana, E., Zhang, X. \& Xia, S. (2018). Quantification of free 2-furfurylthiol in coffee brew using a prefabricated coffee model. Food Anal. Method. 11, 654-662. DOI: 10.1007/s12161-017-1034-8.

3. Sun, Z., Yang, N., Liu, C., Linforth, R.S.T., Zhang, X., \& Fisk, I.D. (2019). Aroma binding and stability in brewed coffee: A case study of 2-furfurylthiol. Food Chem. 295, 449-455. DOI: 10.1016/j.foodchem.2019.05.175.

4. Hofmann, T. \& Schieberle, P. (2002). Chemical interactions between odor-active thiols and melanoidins involve in the aroma staling of coffee beverages. J. Agr. Food Chem. 50, 319-326. DOI: 10.1021/jf010823n.

5. Hofmann, T., Czerny, M., Calligaris, S. \& Schieberle, P. (2001). Model studies on the influence of coffee melanoidins on flavor volatiles of coffee beverages. $J$. Agr. Food Chem. 49, 2382-2386. DOI: 10.1021/jf0012042.

6. Müller, C. \& Hofmann, T. (2007). Quantitative studies on the formation of phenol/2-furfurylthiol conjugates in coffee beverages toward the understanding of the molecular mechanisms of coffee aroma staling. $J$. Agr. Food Chem. 55, 4095-4102. DOI: 10.1021/jf070095p.

7. Sun, Z., Gui, H., Yang, N., Ayed, C., Zhang, X. \& Fisk, I.D. (2020). Enhancement of coffee brew aroma through control of the aroma staling pathway of 2-furfurylthiol. Food Chem. 322, 126754. DOI: 10.1016/j. foodchem.2020.126754.

8. Celebioglu, A., Yildiz, Z.I. \& Uyar, T. (2018). Fabrication of electrospun eugenol/cyclodextrin inclusion complex nanofibrous webs for enhanced antioxidant property, water solubility, and high temperature stability. J. Agr. Food Chem. 66, 457-466. DOI: 10.1021/acs.jafc.7b04312.

9. Yuan, C., Thomas, D.S., Hook, J.M., Qin, G., Qi, K. \& Zhao, J. (2019). Molecular encapsulation of eucalyptus staigeriana essential oil by forming inclusion complexes with hydroxypropyl- $\beta$-cyclodextrin. Food Bioprocess Tech. 12, 1264-1272. DOI: 10.1007/s11947-019-02291-1.
10. Yildiz, Z.I., Celebioglu, A., Kilic, M.E., Durgun, E. \& Uyar, T. (2018). Fast-dissolving carvacrol/cyclodextrin inclusion complex electrospun fibers with enhanced thermal stability, water solubility, and antioxidant activity. J. Mater. Sci. 53, 15837-15849. DOI: 10.1007/ s10853-018-2750-1.

11. Saffarionpour, S. (2019). Nanoencapsulation of hydrophobic food flavor ingredients nanoencapsulation of hydrophobic food flavor ingredients. Food Bioprocess Tech. 12, 1157-1173. DOI: 10.1007/s11947-019-02285-z.

12. Pires, F.Q., Pinho, L.A, Freire, D.O., Silva, I.C.R., SaBarreto, L.L., Cardozo-Filho, L., Gratieri, T., Gelfuso, G.M. \& Cunha-Filho, M. (2019). Thermal analysis used to guide the production of thymol and Lippia origanoides essential oil inclusion complexes with cyclodextrin. J. Therm. Anal. Calorim. 137, 543-553. DOI: 10.1007/s10973-018-7956-6.

13. Ikeda, H., Fukushige, Y., Matsubara, T., Inenaga, M., Kawahara, M., Yukawa, M., Fujisawa, M., Yukawa, E. \& Aki, H. (2016). Improving water solubility of nateglinde by complexation of $\beta$-cyclodextrin. J. Therm. Anal. Calorim. 123, 1847-1850. DOI: 10.1007/s10973-015-4714-x.

14.Zhu, G., Xiao, Z. \& Zhu, G. (2017) Preparation, characterization and release kinetics of mentha-8-thiol3 -one- $\beta$-cyclodextrin inclusion complex. Polym. Bull. 74, 2263-2275. DOI: 10.1007/s00289-016-1835-8.

15. Zhu, G., Jiang, X., Zhu, G. \& Xiao, Z. (2020). Encapsulation of difurfuryl disulfde in $\beta$-cyclodextrin and release characteristics of the guest from its inclusion complex. J. Incl. Phenom. Macro. Chem. 96, 263-273. DOI: 10.1007/s10847-019-00967-x.

16. Zhu, G., Zhu, G. \& Xiao, Z. (2019). A review of the production of slow-release flavor by formation inclusion complex with cyclodextrins and their derivatives. J. Incl. Phenom. Macro. Chem. 95, 17-33. DOI: 10.1007/s10847-019-00929-3.

17.Zhu, G., Feng, N., Xiao, Z., Zhou, R. \& Niu, Y. (2015). Production and pyrolysis characteristics of citralmonochlorotriazinyl- $\beta$-cyclodextrin inclusion complex, $J$. Therm. Anal. Calorim. 120, 1811-1817. DOI: 10.1007/ s10973-015-4498-z.

18. Sun, B. \& Zhang, X. (1993). Study on the synthesis of furfuryl mercaptan. Fine Chemicals, 10, 14-15. DOI: 10.13550/j.jxhg.1993.04.007.

19. Yao, L., Su, C., Qi, L. \& Liu, C. (1999). The substituent structures and characteristic infrared spectra of $\alpha$-furan esters. Spectrosc. Spect. Anal. 19, 32-34. DOI: 10.3321/j.issn:1000-0593.1999.01.011.

20. Yao, L., Su, C., Xu, R., Qi, L. \& Yang, Y. (1998). Synthesis of $\alpha$-furfuryl mercaptan and $\alpha$-furfurylthiol carboxylate and their flavor characteristics. Fine Chemicals, 15, 21-24. DOI: 10.13550/j .jxhg.1998.05.008.

21. Sha, S., Xie, J.C. \& Sun B.G. (2008). Synthesis of flavor furfuryl 2-methyl-3-furyldisulfide. Food Sci. Technol. 5, 140-143. DOI: 10.13684/j.cnki.spkj.2008.05.011.

22.Zhu, G., Xiao, Z., Zhou, R., Zhu, G. \& Niu, Y. (2016). Kinetics and release characteristics of menthyl acetate from its $\beta$-cyclodextrin inclusion complex by thermogravimetric analysis. J. Incl. Phenom. Macro. Chem. 84, 219-224. DOI: 10.1007/s10847-016-0599-y.

23.Zhu, G., Xiao, Z., Zhu, G. \& Rujunzhou, Niu, Y. (2016). Encapsulation of 1-menthol in hydroxypropyl- $\beta$ cyclodextrin and release characteristics of the inclusion complex. Pol. J. Chem. Technol. 18, 110-116. DOI: 10.1515/pjct-2016-0056. 